\title{
Perspectivas alternativas sobre la medición del envejecimiento en España: diferencias territoriales*
}

\author{
Jeroen Spijker \\ Centre d'Estudis Demogràfics \\ jspijker@ced.uab.cat
}

Recibido: septiembre de 2020

Aceptado: noviembre de 2020

Publicado: mayo de 2021

\section{Resumen}

El envejecimiento de la población es el cambio de la estructura por edad en que la proporción de personas mayores aumenta con relación a las más jóvenes. Con frecuencia se considera que este proceso supondrá un incremento importante del gasto público destinado al bienestar y la salud de las personas mayores, razón por la cual el envejecimiento de la población ha servido de justificación para ciertos ajustes de austeridad y el aumento en la edad de jubilación. Mediante cuatro indicadores alternativos, el presente artículo muestra lo siguiente: 1) que el aumento de la supervivencia en edades más avanzadas tiene un efecto rejuvenecedor en la población; 2) que los periodos de auge económico alivian la carga del envejecimiento, mientras que un mercado laboral infrautilizado la agrava. De ahí que los economistas y los responsables de las políticas públicas deban replantearse el uso de los indicadores comunes de dependencia de la vejez basados en la edad de 65 años como "umbral de vejez» y la edad de 16 a 64 como la población "productiva» para justificar sus argumentos y políticas.

Palabras clave: envejecimiento de la población; tasas de dependencia en la vejez; esperanza de vida; gasto público; España; análisis territorial

Resum. Perspectives alternatives sobre el mesurament de l'envelliment a Espanya: diferències territorials

L'envelliment de la població és el canvi de l'estructura per edat de la població en què la proporció de persones grans augmenta amb relació a les més joves. Sovint es considera que aquest procés suposarà un increment important de la despesa pública destinada al benestar i la salut de les persones grans, raó per la qual l'envelliment de la població ha servit de justificació per a certs ajustos d'austeritat i l'augment en l'edat de jubilació. Mitjançant quatre indicadors alternatius, el present article mostra el següent: 1) que l'augment de la

* Este trabajo se enmarca en los siguientes proyectos: «¿Las personas mayores tendrán parientes que les podrán cuidar en el futuro? Un estudio basado en un modelo mixto de micro-simulación y en agentes» (CSO2017-89721-R; co-IP J Spijker), financiado por el Plan Nacional I+D+i del Ministerio de Ciencia e Innovación, el programa Ramón y Cajal (RYC-201314851; IP J Spijker), financiado por el mismo ministerio, y el proyecto «HEALIN», financiado por el European Research Council (ERC-2019-CoG-864616; IP Iñaki Permanyer). 
supervivència en edats més avançades té un efecte rejovenidor en la població; 2) que els períodes d'auge econòmic alleugen la càrrega de l'envelliment, mentre que un mercat laboral infrautilitzat l'agreuja. D'aquí que els economistes i els responsables de les polítiques públiques han de replantejar-se l'ús d'indicadors comuns de dependència de la vellesa basats en l'edat de 65 anys com a «llindar de vellesa» i l'edat de 16 a 64 com la població "productiva» per justificar els seus arguments i polítiques.

Paraules clau: envelliment de la població; taxes de dependència en la vellesa; esperança de vida; despesa pública; Espanya; anàlisi territorial

Résumé. Perspectives alternatives sur la mesure du vieillissement de la population en Espagne: différences territoriales

Le vieillissement de la population est le changement de la structure par âge dans laquelle la proportion de personnes âgées augmente par rapport aux plus jeunes. On considère souvent que ce processus conduira à une dépense publique de plus en plus importante afin de maintenir le bien-être et la santé des personnes âgées, raison pour laquelle le vieillissement de la population a permis de justifier des mesures d'austérité, ainsi que par le recul de l'âge de départ à la retraite. Au moyen de quatre indicateurs alternatifs, cet article montre que 1. l'augmentation de la survie à un âge avancé a un effet rajeunissant sur la population; 2 . les essors économiques allègent le fardeau économique du vieillissement, tandis qu'un marché du travail sous-utilisé l'aggrave. Par conséquent, les économistes et les responsables publics devraient reconsidérer l'utilisation des indicateurs communs de dépendance des personnes âgées basés sur l'âge de 65 ans comme " seuil de la vieillesse " et de 16 à 64 ans en tant que population "productive" pour justifier leurs arguments et leurs politiques.

Mots-clés : vieillissement de la population; taux de dépendance des personnes âgées ; espérance de vie ; dépenses publiques ; Espagne ; analyse territoriale

Abstract. Alternative perspectives on measuring population ageing in Spain: Regional differences

Population ageing is the change in a population's age structure in which the proportion of older people increases relative to younger people. It is often considered that this process leads to a significant increase in public spending on elderly welfare and health, which explains why population ageing has served to justify certain austerity measures as well as to increase the retirement age. Using four alternative indicators, this article shows that 1) increased survival at older ages has a rejuvenating effect on the population and 2) economic booms ease the burden of ageing, while an underutilized labor market exacerbates this burden. Hence, economists and public policy makers should rethink the use of common old-age dependency indicators based on age 65 as the "threshold of old age" and age 16-64 as the "productive" population to justify their arguments and policies.

Keywords: population aging; old-age dependency rates; life expectancy; public spending; Spain; regional analysis

\section{Sumario}

$\begin{aligned} \text { 1. Introducción } & \text { 4. Resultados } \\ \text { 2. Metodología } & \text { 5. Discusión } \\ \text { 3. Datos } & \text { Referencias bibliográficas }\end{aligned}$




\section{Introducción}

El envejecimiento — causado por unos niveles permanentes de fecundidad inferiores al nivel de reemplazo y una reducción de la mortalidad en las edades avanzadas - ha conducido a un aumento inexorable de la proporción de personas mayores de 65 años, y principalmente del grupo de mayores de 80 años, en los países desarrollados, y también en España. Este proceso de envejecimiento, que se acompaña de un aumento de la proporción de personas dependientes por razón de salud, se ha convertido en una de las mayores preocupaciones de los políticos por sus implicaciones presentes y futuras sobre la productividad del trabajador y la viabilidad de los sistemas de jubilación, salud y de protección social (Doyle et al., 2009; Polder et al., 2002; Wolf y Amirkhanyan, 2010). Sin embargo, el análisis de este proceso se está reconsiderando en la actualidad, debido a la constatación de que los indicadores de envejecimiento tradicionales no son adecuados (Sanderson y Scherbov, 2010). Es lo que se ha llamado la "paradoja del envejecimiento»: sus factores causantes explican a la vez por qué las personas de una determinada edad de hecho "rejuvenecen", debido a que el número de años de esperanza de vida a esta edad es mayor que el de las generaciones anteriores. Esta paradoja se debe a que la población puede envejecer (su edad mediana aumenta) y a la vez rejuvenecer (la esperanza de vida a esta edad mediana aumenta), tal como se observó en España entre 1973 y 1981 (figura 1). A partir de ahí, podría argumentarse que, en periodos de rápido descenso de la mortalidad, la edad de las personas no es la métrica adecuada para medir el cambio en la estructura por edad de una población.

El concepto de «años vividos» (la edad cronológica o retrospectiva) no es más relevante que el de «años que quedan por vivir» (la edad prospectiva). Aunque estas ideas no son nuevas (Ryder, 1975; Sanderson y Scherbov, 2005, 2007, 2010; Spijker y MacInnes, 2013), no existen estudios en profundidad sobre España. Esto es así a pesar de su utilidad teórica y práctica para las administraciones públicas. En efecto, se podría tener una mejor comprensión de cómo los cambios en la estructura de la población, las mejoras en la salud de las personas y la coyuntura económica influyen en la productividad económica y los ingresos fiscales obtenidos, los cuales son necesarios para mantener los sistemas del bienestar social y de salud. Sin embargo, valga mencionar varias publicaciones de economistas y demógrafos españoles que ya han cuestionado el uso de indicadores tradicionales para la medición de la dependencia demográfica y la vejez. Por ejemplo, Fernández Cordón $(1996,2015,2017)$ y Blanes et al. (1996) los criticaron por la falta de consideración de la dinámica del mercado laboral. Según los autores del último trabajo citado (enmarcado en el contexto de la década de 1990), «el aumento de la población en edad de trabajar atribuible a los nacidos en los años de elevada natalidad no ha supuesto ninguna ventaja $\mathrm{y}$, en cambio, se ha traducido en preocupantes porcentajes de inactividad y de paro" (ibid.: 150-151).

En un marco de pensamiento similar, la Autoridad Independiente de Responsabilidad Fiscal (AIReF) (2019) mostró más recientemente, mediante ejercicios proyectivos, que la viabilidad del estado de bienestar y del sistema 
Figura 1. La edad mediana (EM) y la esperanza de vida restante (EVR) a dicha edad mediana, 1950-2018

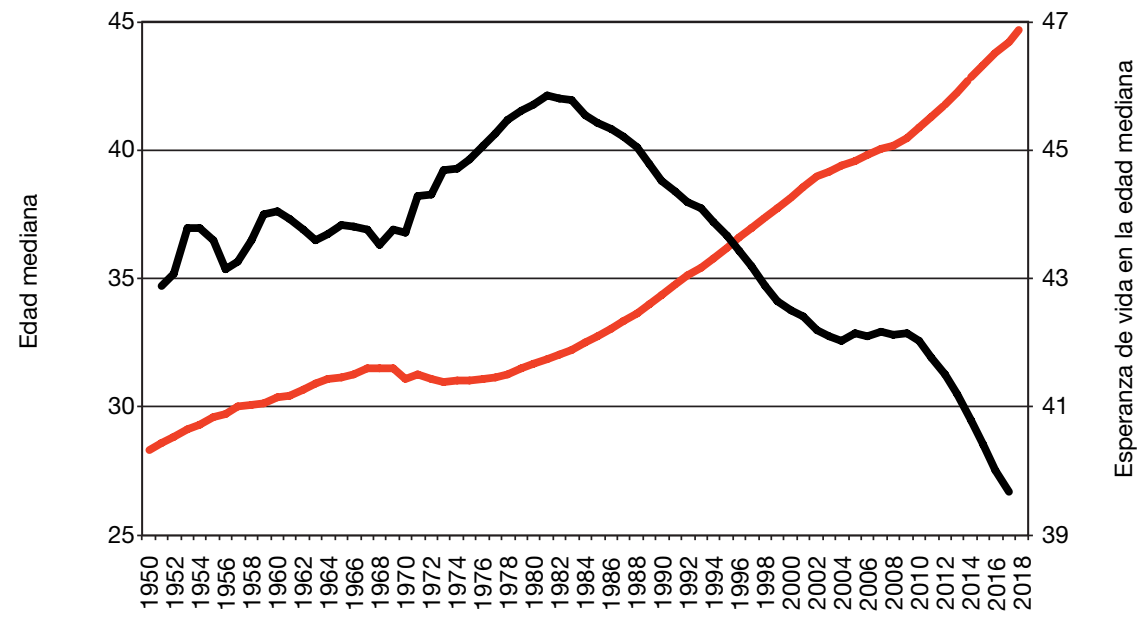

- Edad mediana (EM) - EVR en la EM (promedio 3 años)

Fuente: elaboración propia a partir de los datos del Human Mortality Database (<www.mortality.org >).

de salud pública está más relacionada con la participación laboral, el nivel de productividad y el sistema de tributación de un país que con el envejecimiento demográfico. Finalmente, Blanes y Cabré (2015), que también consideraron necesario replantearse el propio concepto de envejecimiento demográfico por los avances en la longevidad, proyectaron la población mayor según tres criterios de edad: la edad cronológica (65 y más), la edad prospectiva (basada en años restantes) y la edad equivalente en buena salud. Según sus resultados, habrá 2 millones de personas "mayores» menos en 2064 que cuando se contabiliza la población de 65 y más, si se considera no solo la edad sino también el nivel de salud de la población.

El indicador estándar del envejecimiento de la población es la tasa de dependencia en la vejez (TDV) (en inglés, old age dependency ratio). Toma el número de personas que han alcanzado la edad de 65 años y lo divide por el número de adultos en «edad laboral» (que generalmente abarca la edad 16-64 o 20-64). Supuestamente, mide la población dependiente de edad avanzada en relación con aquellos que financian su bienestar:

$$
\mathrm{TDV}=\left(\frac{\sum \text { población } 65+}{\sum \text { población } 16-64}\right) \times 100
$$

Sin embargo, la TDV no es el indicador más adecuado, ya que no cuenta correctamente ni los a ancianos dependientes ni a quienes los sostienen. Sim- 
plemente toma un punto de corte (la edad teórica de jubilación) y asigna, en consecuencia, a los adultos a los dos lados de la proporción. Por este motivo, en este artículo se presentarán para España — tanto en el ámbito estatal como provincial y con una breve comparación internacional — varios indicadores prospectivos de envejecimiento alternativos a la proporción de la población mayor de 65 años y la TDV. Estos indicadores alternativos se adaptan mejor a los cambios en la mortalidad, así como a las fluctuaciones económicas, procesos importantes para la política pública, sobre todo en relación con el sistema de pensiones y la salud. Los resultados muestran que, después de corregir por las mejoras en la esperanza de vida en edades avanzadas, la tasa de dependencia en España no aumentó desde 1950 y se prevé que solo aumente ligeramente a partir de 2020.

\section{Metodología}

El punto de partida de los debates sobre envejecimiento de la población suele estar equivocado, porque se basa en una definición de «edad avanzada» fija, generalmente a partir de la edad de 60 o 65 años. Esto lleva a infravalorar los efectos de las mejoras continuas en la mortalidad en edades avanzadas como resultado de los progresos médicos y de la reducción del ritmo del envejecimiento biológico. Estos factores explican por qué muchas personas de más de 65 años tienen mejor estado de salud y vivirán más años que las personas que alcanzaron esta edad en un pasado reciente. En este sentido, «vejez» y «edad» deberían diferenciarse en el terreno conceptual y metodológico, ya que no tienen una relación unívoca. Construir distintos indicadores del envejecimiento poblacional basados solamente en una edad fija es engañoso (Lutz et al., 2008), porque esto asume implícitamente que no habrá progresos en factores importantes como la esperanza de vida restante (EVR) a esta edad y los riesgos de discapacidad. A la inversa, medidas ajustadas por el cambio en la longevidad muestran una tendencia de cambio menor en el tiempo que las basadas en la definición tradicional de edad. Por este motivo, Sanderson y Scherbov (2007, 2010) proponen un nuevo enfoque para medir la edad: la orientación hacia el futuro (forward-looking). El punto central de esta nueva perspectiva es establecer la existencia de dos edades diferentes para cada persona (Brouard, 1989; Vaupel, 2009; Villavicencio y Riffe, 2016): 1) los años vividos (la edad cronológica o retrospectiva), es decir, todos aquellos que tengan la misma edad han vivido el mismo número de años; 2) la edad prospectiva, con una perspectiva de futuro. Eso implica que todos aquellos con la misma edad prospectiva tienen por delante la misma expectativa de años por vivir (es decir, la EVR).

En la tabla 1 se muestra cómo calcular la edad prospectiva. El panel de la izquierda contiene dos columnas de la tabla de mortalidad para una persona de edad en un determinado año, que recibe el nombre de año indice, en este caso 2016. En la primera columna se incluye la edad de la persona $(a)$ y en la segunda se muestra la EVR a esa edad, también en el año indice. El panel de la derecha contiene las mismas dos columnas de las tablas de mortalidad, 
Tabla 1. Determinación de la edad prospectiva

\begin{tabular}{|c|c|c|c|}
\hline Tabla de mortalidad & o índice (2016) & Tabla de mortalidad & estándar (1950/197 \\
\hline Edad retrospectiva & EVR & EVR & Edad prospectiva \\
\hline$a$ & & & $A$ \\
\hline p. ej. 40 años & 44 años & 44 años (1950) & 30 años \\
\hline p. ej. 73 años & 15 años & 15 años (1970) & 65 años \\
\hline
\end{tabular}

Fuente: elaboración propia a partir de los datos del Human Mortality Database (<www.mortality.org >).

pero están presentadas en sentido opuesto. La primera columna de este panel contiene la EVR de la edad $a$, pero esta vez en la tabla de mortalidad del año con el que se quiere hacer una comparación (año estándar). Por último, la siguiente columna del panel contiene la edad exacta en la que el año estándar tenía la misma EVR que en la segunda columna. La edad prospectiva de una persona con edad $a$ en el año índice es la edad en el año estándar denotada como $A$. Entonces, la EVR de la persona de edad $a$ en el año índice es la misma que la de una persona de edad $A$ en el año estándar (Sanderson y Scherbov, 2007). En el ejemplo de arriba, la edad prospectiva de alguien de 40 años en 2016 es 30 años si usamos 1950 como estándar (ya que la EVR en estas edades es igual). Del mismo modo, a personas en edad legal de jubilación en 1970 (tomado aquí como año estándar) les quedaban, de promedio, 15 años por delante, igual que para las personas de 73 años en 2016. En este caso, 65 es la edad prospectiva para personas de 73 años: es como si una persona de 73 años tuviera la misma edad que una persona de 65 años en 1970, puesto que las dos tenían la misma esperanza de vida. Estimaciones del Instituto Nacional de Estadística (INE) sugieren que en 2050 la edad en que la EVR será igual a 15 años será a los 76 años (ver figura 2). Una pregunta clave es si podemos asumir que personas con la misma EVR estén igual de envejecidas a pesar de sus diferentes edades. Según la literatura, muchos tipos de comportamiento y actitudes, incluso los relacionados con la salud, el ahorro, la actividad laboral y el consumo, están más vinculados con la esperanza de vida que le queda a una persona que con su edad retrospectiva (Bíró, 2013; Carstensen, 2006; Carstensen et al., 1999; Cocco y Gomes, 2012; Hamermesh, 1985; Van Solinge y Henkens, 2010; Wong et al., 2011).

Aplicando este enfoque prospectivo mediante la EVR, se pueden obtener indicadores alternativos a la TDV para medir el envejecimiento de una población. Uno es la tasa prospectiva de la dependencia en la vejez (TPDV):

$$
\operatorname{TPDV}=\left(\frac{\sum_{s} \text { población } E V R \leq 15}{\sum_{s} \text { población de } 20+\text { años hasta la edad donde EVR }>15}\right) \times 100
$$

La TPDV tiene como numerador la suma de hombres y mujeres (estimada por separado, como indica la letra $s$ en la fórmula) que poseen una EVR de 15 
Figura 2. Umbral móvil de la edad exacta a la que restan 15 años de esperanza de vida. España, 1950-2050

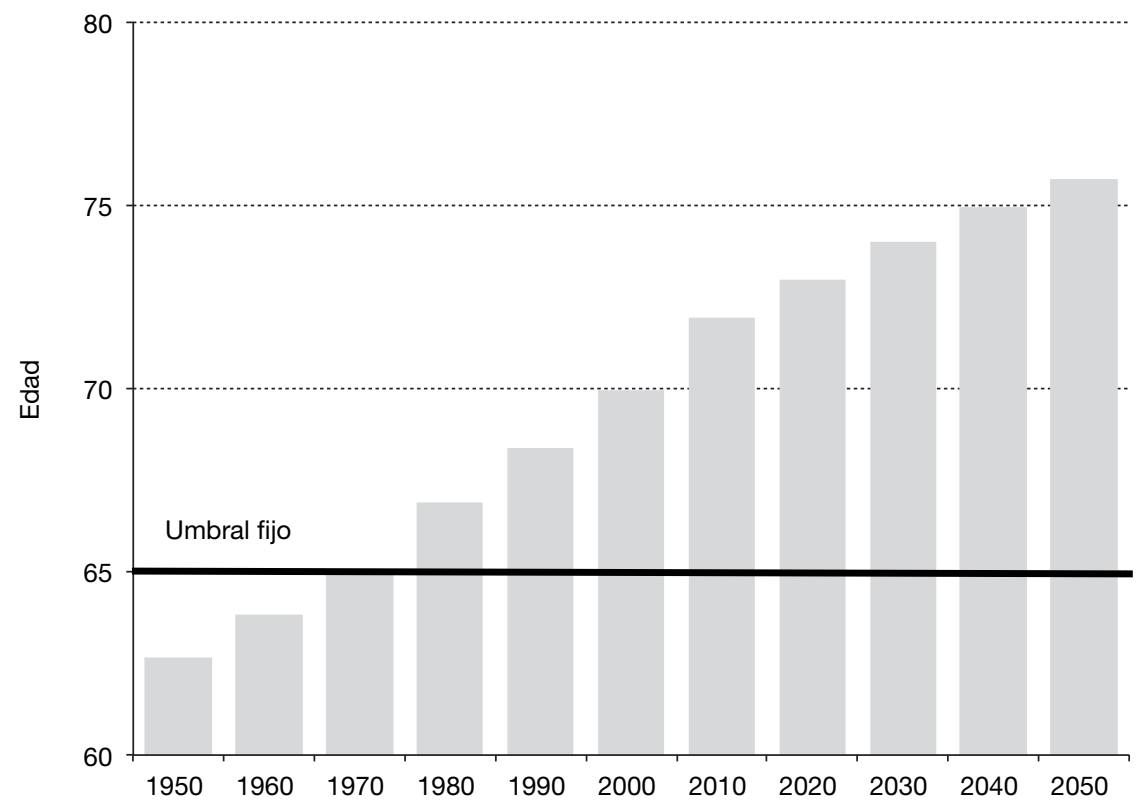

Fuente: Human Mortality Database y las proyecciones de población del INE (ver sección de datos para más detalle).

años o menos, en lugar del total de personas de 65+ años, como sucede con la tasa de dependencia tradicional (TDV). El denominador es el número de adultos hombres y mujeres que tengan desde los 20 años $^{1}$ de edad hasta la edad con una EVR superior a 15 años. Esta se considera la población que potencialmente puede sostener económicamente a la población de edad avanzada según la nueva definición (Sanderson y Scherbov, 2007).

A partir de aquí, se puede mejorar aún más el indicador teniendo en cuenta la actividad económica, puesto que una parte importante de la población en edad laboral no participa en el mercado laboral. Así surgió la «tasa real de dependencia en la vejez» (TRealDV, en inglés real elderly dependency ratio), en la que se limita la población del denominador a las personas que tienen un empleo remunerado y así contribuyen económicamente a mantener los sistemas del bienestar (por ejemplo, las pensiones) y de salud a través de sus cotizaciones e impuestos (Spijker y MacInnes, 2013):

1. En lugar de 15 y 16 que se suele usar como umbral para la TDV, ya que muchos adolescentes mayores y adultos jóvenes todavía asisten a la educación secundaria o superior, lo que significa que la mayoría aún no son (totalmente) activos laboralmente. 


$$
\text { TRealDV }=\left(\frac{\sum_{s} \text { población EVR } \leq 15}{\text { Población con empleoremunerado }}\right) \times 100
$$

Sin embargo, hay mucha diferencia entre sectores económicos según la productividad y el valor del producto. Además, la productividad económica puede variar en el tiempo por razones ajenas a cambios en el tamaño de la fuerza del trabajo. Dada la importancia de la coyuntura económica (tiempos de crisis y crecimiento económico), el producto interior bruto (PIB) es un indicador que puede captar la capacidad potencial del conjunto de la economía de cubrir los gastos en salud y bienestar de las personas (mayores) dependientes. De ahí, el siguiente indicador, que capta la productividad por cada adulto mayor, ajustada por los cambios en la esperanza de vida:

$$
\text { Tasa de personas mayores a } \mathrm{PIB}(\mathrm{TPMPiB})=\frac{\sum_{s} \text { población } E V \leq 15}{P I B}
$$

donde el numerador consiste en el PIB en US\$ en paridad de poder adquisitivo (PPA) en precios actuales (2018), actualizados al nivel de 2011 (The Conference Board, 2019).

Finalmente, dado que cualquier gasto del Gobierno en las personas mayores debe proceder de los impuestos, aunque una proporción importante (casi una quinta parte) de la economía española es sumergida (Schneider, 2016), otra tasa alternativa a la TDV es utilizar los ingresos fiscales obtenidos por el Gobierno como denominador alternativo (Spijker, 2015):

Tasa de personas mayores a impuestos $(\mathrm{TPMI})=\frac{\sum_{s} \text { población } E V \leq 15}{\text { impuestos }}$

\section{Datos}

Disponemos de buenos datos sobre las condiciones de salud, los cuales son más relevantes para analizar las condiciones de vida de las personas mayores que los datos de mortalidad. El problema es que no existen series temporales largas. Además, las comparaciones temporales se ven dificultadas por cambios sucesivos en la redacción de las preguntas incluidas en diferentes ediciones de encuestas de salud (Berger y Van Oyen, 2013). Un buen sustituto son las series largas de la EVR calculadas a partir de las tablas de mortalidad anuales. Dan una visión más exacta del grado de envejecimiento al tener en cuenta el descenso de los riesgos de mortalidad a edades avanzadas, para lo cual se utiliza un umbral de edad variable. En efecto, el comienzo de la edad de vejez está definido por una situación de dependencia constante y no un límite de edad constante: en vez de la edad 65, se usa la edad con un nivel de EVR igual a 15 
años. Esta edad ha aumentado de forma constante en los países desarrollados desde los años 1960, lo que limita el aumento de la tasa de dependencia prospectiva (Lutz et al., 2008; Sanderson y Scherbov, 2010; Spijker y MacInnes, 2013). También en casi todos los países de América Latina se ha mostrado que la TPDV experimenta caídas significativas que se prolongan hasta la década de 2020 y que llegan a alcanzar niveles inferiores a los observados en el pasado, mientras que la tasa de dependencia tradicional, la TDV, no deja de crecer (Rivero-Cantillano y Spijker, 2015).

La EVR para España y los efectivos de la población por edad simple y sexo están disponibles desde 1950 hasta 2018 en el Human Mortality Database (<www.mortality.org>), y para las proyecciones hasta 2050 en la página web del INE ${ }^{2}$. Los datos de empleo requeridos para el denominador de la TRealDV provienen de la Encuesta de la Población Activa (EPA) para el análisis por provincias ${ }^{3}$, y de la Organización para la Cooperación y el Desarrollo Económicos (OCDE) para el análisis nacional y el internacional (OECD, 2020). Los datos sobre el PIB en España provienen del Conference Board Total Economy Database (The Conference Board, 2019) y los datos de impuestos provienen de la OCDE (OECD, 2019).

\section{Resultados}

\subsection{Resultados en el ámbito nacional}

El nivel y la tendencia de estos nuevos indicadores son muy diferentes que los de la ratio de dependencia tradicional, al menos entre 1950 y 2030 , tal como se puede apreciar en la figura 3. En el caso de la TPDV, la tasa de dependencia es casi igual en la actualidad (17,3\% en 2019) que en 1950 (17,9\%) y en 1970 $(16,4 \%)$. En comparación, si calculamos la tasa de dependencia de la manera tradicional (TDV), la tasa era del 30,2\% en 2019, casi el doble que en 1970 $(15,7 \%)$ y casi tres veces más que en $1950(11,1 \%)$. Si además de controlar por las mejoras en la EVR (es decir, considerando como umbral de vejez la edad donde la esperanza de vida es igual a 15 años) también incluimos los cambios en la participación laboral (ya que son los trabajadores los que finalmente pagan el sistema de salud y las pensiones), vemos que hasta recientemente la dependencia real (TRealDV) estaba por encima de la TDV, pero, debido al

2. <https://www.ine.es/dynt3/inebase/index.htm?padre=6671\&capsel=6674>. Proyección de la población. Resultados nacionales: serie 2018-2067. 1.1.1.2 Población residente en España a 1 de enero, por sexo, edad y año y 1.1.2.2.3 Tablas de mortalidad por sexo, edad, funciones y año (descargados 4-8-2020). Resultados provinciales: serie 2014-2028. 1.3.1.2 Población residente en España a 1 de enero, por sexo, edad y año y 1.3.2.2.3 Tablas de mortalidad por sexo, edad, funciones y año (descargados 1-8-2016). Actualmente, las estimaciones de esperanza de vida por provincias están solamente disponibles para edades quinquenales, razón por la cual se decidió trabajar con las estimaciones anteriores, ya que los datos con intervalos por edad simple son más precisos y la esperanza de vida no es un fenómeno demográfico que muestra importantes cambios anuales en España.

3. <https://www.ine.es/jaxiT3/Tabla.htm?t=4076>. 
Figura 3. La tasa de dependencia en la vejez (TDV), la tasa prospectiva de la dependencia en la vejez (TPDV), la tasa real de dependencia en la vejez (TRealDV) y la TRealDV ajustada por un aumento en la participación laboral (TRealDVempleo+). España, 1950-2050

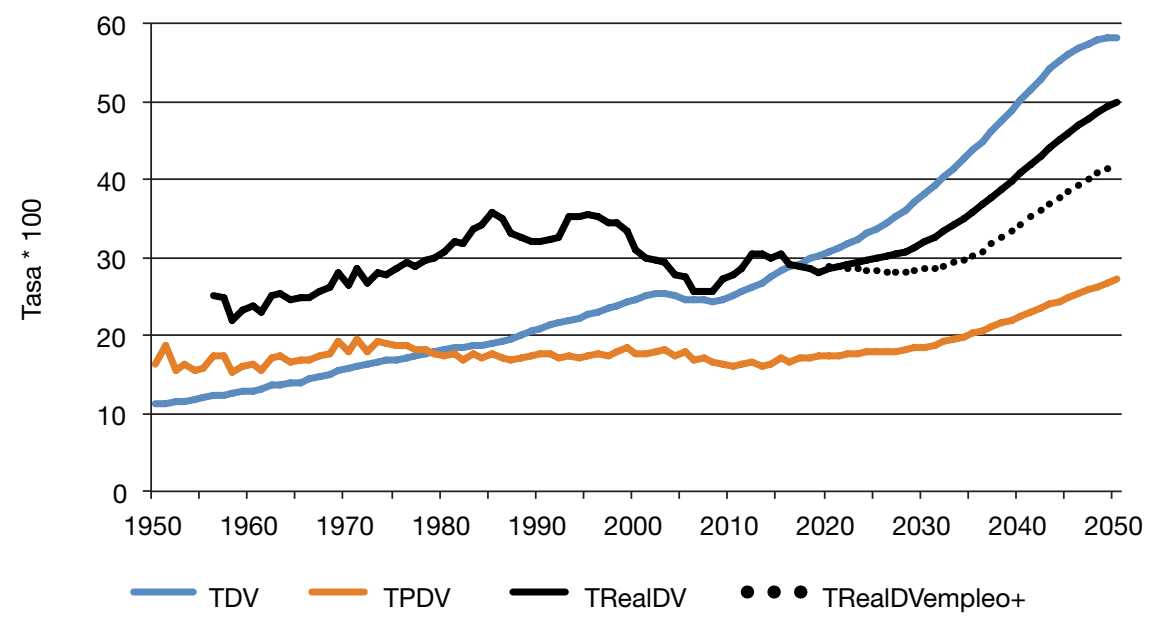

Nota: En cuanto a la TRealDVempleo+, se supone un aumento lineal en la participación laboral entre las personas de 16-64 del 64\% actual (Q1 2020) al 67\% en 2025 (el nivel de 2007, el año en que la tasa de empleo era la más alta en medio siglo) y hasta el 77\% a partir de 2035 (el nivel actual en Japón, Nueva Zelanda y Suecia), para la edad 65-69 años un aumento desde el 7\% actual (Q1 2020) al 9\% en 2025 (extrapolación del aumento entre 2015 y 2020) y hasta el $12 \%$ a partir de 2035, y para las personas de $70+$ desde el $0,94 \%$ en 2020 al $1 \%$ en 2025 y hasta el $2 \%$ en 2035 (todavía muy lejos de las tasas de empleo entre personas mayores de 70 años en países como Nueva Zelanda, donde en 2017 el $13 \%$ seguía trabajando (Maré, 2018)). Fuente: elaboración propia a partir de los datos del Human Mortality Database, la EPA y las proyecciones de población del INE (ver sección de datos para más detalle).

Fuente: elaboración propia a partir de los datos del Human Mortality Database, la EPA y las proyecciones de población del INE (ver sección de datos para más detalle).

aumento en la EVR entre las personas mayores y la entrada masiva de mujeres en el mercado laboral, la ratio bajó desde los años 80 hasta el comienzo de la anterior crisis económica (2008-14). Ahora, si la tasa de empleo para la población en edad laboral (no de actividad, ya que en 2019 un 14\% de la población siguió estando parada según los datos de la EPA, y es, por tanto, dependiente para el Estado o para sus familiares igual que los inactivos) subiese a niveles de justo antes del comienzo de la crisis de 2008-14 y con aumentos moderados en la actividad entre la población mayor de 65 años (ver nota bajo la figura 3), la TRealDV no cambiaría en absoluto hasta 2031.

Finalmente, reemplazando los ocupados por a) el PIB y b) los impuestos obtenidos por el Estado, se puede observar que la tendencia en el número real de personas mayores (es decir, contabilizadas según nuestra definición) por cada US\$ 1 millón producido e impuestos fiscales recaudados no parece alarmante. Ambas tendencias son similares (figura 4). En cuanto al primer indicador, en 1965 había 9,0 personas mayores por US\$ 1 millón de PIB producido, pero tan solo 3,0 personas en 2007. Aunque dicha tasa subió ligeramente durante la crisis (3,3 en 2012), en 2019 la tasa había bajado justo por debajo 
Figura 4. La tasa de personas mayores a PIB (TPMPIB), la tasa de personas mayores a impuestos (TPMI) y ambos con un crecimiento del $2 \%$ en vez del 1\% a partir del 2031. España, 1950-2050

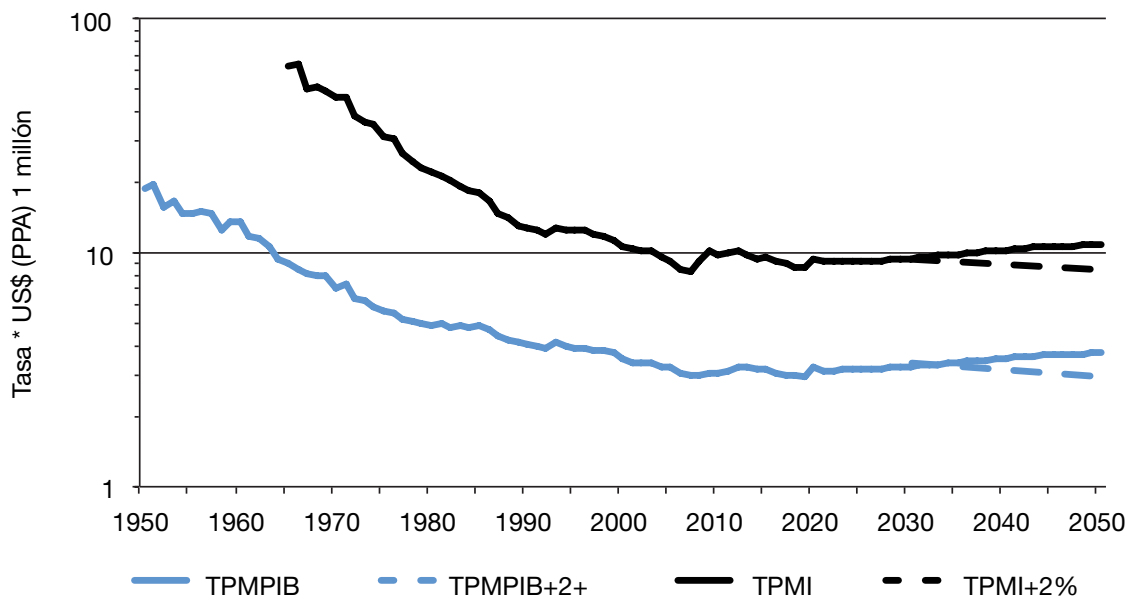

Fuente: elaboración propia a partir de los datos del Human Mortality Database, la OECD (2020), The Conference Board (2019) y las proyecciones de población del INE (ver sección de datos para más detalle).

de $3(2,95)$, y aunque se predice que la crisis económica actual causada por la COVID-19 provocará un ligero aumento en el número de personas mayores por US\$ 1 millón de PIB (3,05 en 2020), la predicción es que la «carga económica» de las personas mayores seguirá bajando ligeramente por las mejoras en la productividad económica una vez que se haya superado la pandemia. De forma semejante, el número de personas mayores por US\$ 1 millón de impuestos recaudados bajó substancialmente entre 1965 y 2006, de las 63,0 personas mayores a 8,3. Cabe destacar que entre principios de los setenta y el año 2000 el total de impuestos fiscales como proporción del PIB se duplicó con creces del $16 \%$ al 33\%. Si proyectamos los impuestos recaudados en el futuro sobre la base de un crecimiento de $-8,0 \%$ en $2020,+4,3 \%$ en $2021,+0,7 \%$ entre 2022 y 2030 (igual al crecimiento previsto en el PIB por el IMF (2020)) y $+1,0 \%$ hasta 2050 (tomado de las previsiones del The Economist Intelligence Unit (2020)), se observa que la carga económica aumentará ligeramente puesto que el crecimiento económico se queda por detrás del aumento del envejecimiento demográfico. Sin embargo, si subimos la tasa de crecimiento económico al $2 \%$ anual, la carga económica permanece muy estable en el tiempo.

\subsection{Resultados en el ámbito internacional}

Internacionalmente, España es uno de los países más envejecidos según la TDV (figura 5). No obstante, durante los años de boom económico, la TRealDV bajó en picado, en parte por la llegada de millones de inmigrantes en el mercado 
Figura 5. La tasa de dependencia de la vejez (TDV) y la tasa real de dependencia de la vejez (TRealDV). Varios países, 1950-2018 (3 años media móvil)

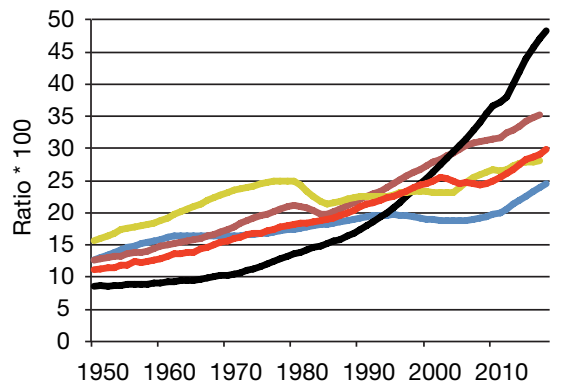

EE.UU. Italia Japón

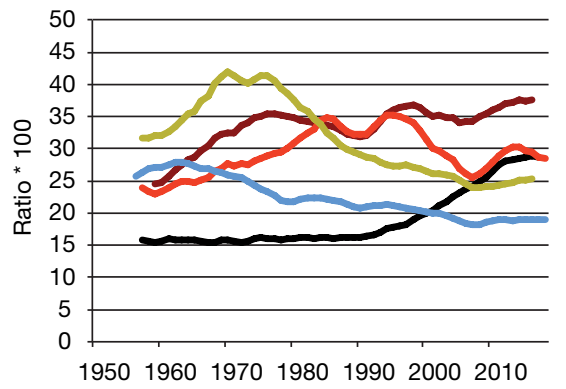

Austria España

Fuente: elaboración propia a partir de los datos del Human Mortality Database y The Conference Board (2019) (ver sección de datos para más detalle). Nota: Gráfico de la izquierda: TDV - 1950-2018 para todos los países. Gráfico de la derecha: TRealDV - 1956-2018 para España y EE. UU., 1958-2017 para Italia, y 1950-2017 para Austria y Japón.

laboral, pero también por las mejoras en la esperanza de vida a edades avanzadas. Sin embargo, desde 2008 la TRealDV ha vuelto a crecer, de hecho, a un ritmo más intenso que la TDV normal debido a la reducción de 3,4 millones de puestos de trabajo entre 2007 y 2013. La mayoría de los otros países también han tenido un rebote en la tendencia, pero mucho menor porque el paro subió menos. Por ejemplo, con relación a los ejemplos mostrados en la figura 4, la tasa de paro en Austria subió del 4\% en 2008 al 6\% en 2016 (<http://stats.oecd. org $>$ ). Es interesante señalar que Japón, el país más envejecido del mundo visto desde la perspectiva tradicional, tiene una TRealDV más baja que España. Esto se debe parcialmente a que su EVR a edades avanzadas es todavía ligeramente más alta, pero lo más importante es que tienen una alta tasa de empleo $(78 \%$ en 2019), causada por una muy baja tasa de paro (2,3\% en 2019). Por otro lado, los EE. UU. tienen una de las TRealDV más bajas de los países desarrollados, no por tener una EVR tan alta como Japón, sino porque tienen una población más joven y también, como en el caso de Japón, una tasa alta y creciente de empleo (71\% en 2019), gracias a que se recuperaron de la crisis antes que España. Finalmente, un país que está peor situado que España, también según el indicador alternativo de envejecimiento, es Italia, básicamente debido a un mayor envejecimiento y una tasa de empleo inferior a la de España.

\subsection{Resultados en el ámbito territorial}

A pesar del alto nivel de esperanza de vida, hay claras diferencias territoriales en España en la edad donde la EVR es igual a 15 años. Como se puede observar en la figura 6a, esa edad es baja en las provincias del sur del país, en la Comunidad Valenciana y las islas Baleares, así como en Asturias, Cantabria y Las 
Palmas, que corresponden a los territorios con esperanzas de vida más bajas de España. Al contrario, las edades más altas se concentran en las provincias de Castilla y León, Madrid, Navarra y la mayoría de las provincias de Castilla-La Mancha, Aragón y el País Vasco. Es interesante ver que los patrones territoriales de este umbral de la vejez son similares para ambos sexos (tal vez con la excepción del País Vasco; ver las figuras $6 \mathrm{~b}$ y $6 \mathrm{c}$ ). Esta constatación no es nueva (p. ej., ver Blanes, 2007), pero lo interesante es que en este caso lo estamos mirando al revés, es decir, analizando la edad del umbral de la vejez, aquí considerada cuando la EVR es igual a 15 años (como en Sanderson y Scherbov, 2007; 2010), en vez de la EVR a edad de 65 (como en Blanes, 2007).

Si contamos el número de personas en estas edades (considerado como la población dependiente) y lo dividimos por los adultos con más de 15 años de esperanza de vida, obtenemos la tasa prospectiva de dependencia en la vejez (TPDV) (figura 7). De nuevo, varias provincias de Castilla y León tienen valores altos, pero también Asturias y las provincias de Galicia. Por otro lado, las provincias de la costa de Andalucía más Sevilla y Murcia, así como Madrid, Guadalajara, las islas Baleares y las Canarias, tienen las TPDV más bajas (figura 7a).

Las figuras $7 \mathrm{~b}$ y $7 \mathrm{c}$ dan las tasas por sexo. Lo llamativo de este perspectivo de vejez es que las mujeres apenas están sobrerrepresentadas. Eso es porque la EV en edades avanzadas es más alta entre las mujeres, y por tanto el umbral de la vejez (la edad donde la EVR=15 años) es más elevado, pero a su vez corresponde a generaciones menos numerosas. Aunque se suele asumir que haya muchas más mujeres mayores que hombres, eso es porque se está comparando personas de la misma edad cronológica (en 2019 habían 30\% más mujeres que hombres de 65 años o más). En cambio, si lo miramos desde la perspectiva de la edad prospectiva (por ejemplo, asumir que una mujer de 74 años está tan envejecida como un hombre de 70), el exceso de mujeres es mucho menor (7\%).

Volviendo a los resultados y utilizando la misma escala para ambos sexos, se puede observar que las mujeres cuentan con varias provincias más en la categoría de mayor nivel de envejecimiento (Cuenca, Palencia y Segovia), así como en el segundo mayor nivel (destacan las cuatro provincias contiguas del sur de país, Badajoz, Ciudad Real, Córdoba y Jaén), pero las diferencias absolutas son pocas.

Al comparar el patrón regional de la TPDV para la población total (figura 7a) con el indicador clásico - la TDV - para la población total (figura 8a), apenas se observan diferencias, aunque el valor absoluto de la TDV es bastante más alto que el de la TPDV. Además, ahora sí, la población femenina es mucho más envejecida que la masculina cuando se comparan los dos sexos (figuras $8 \mathrm{~b}$ y 8c). Eso se explica por su mayor esperanza de vida a cada edad desde los 65 años (el umbral fijo para este indicador), y como consecuencia hay más mujeres en estas edades ${ }^{4}$. Pero si una mujer de 74 años tiene la misma EVR que

4. Además, en cuanto al denominador de la TDV, hay más hombres, mientras que el umbral en la TPDV, que está bastante por encima de los 65 años, en total hay más mujeres que hombres mayores de 20 años con una EVR de 15 años o más. 
Figura 6. La edad en que la esperanza de vida (EV) es igual a 15 años por provincias en España, 2019

a) Total

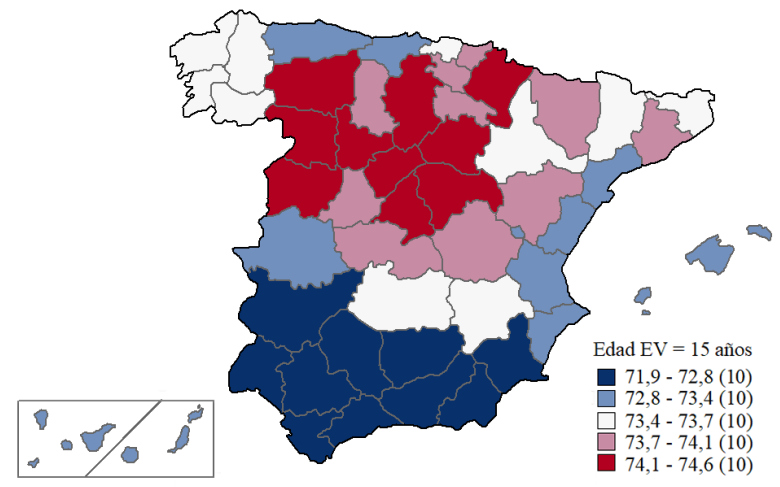

b) Hombres

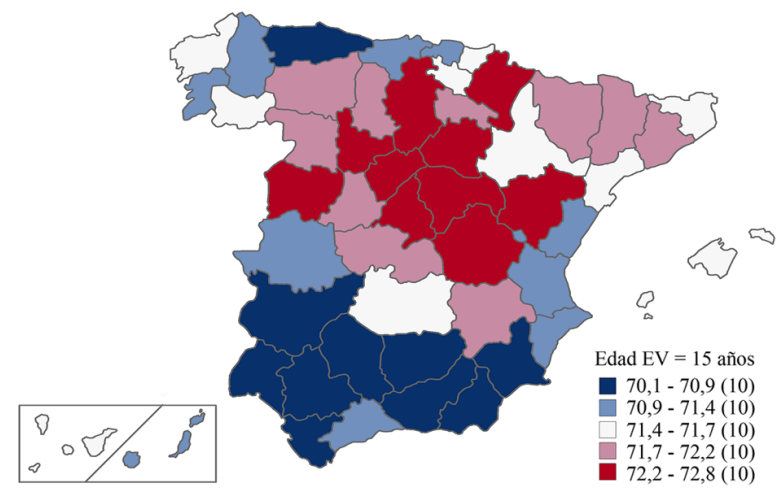

c) Mujeres

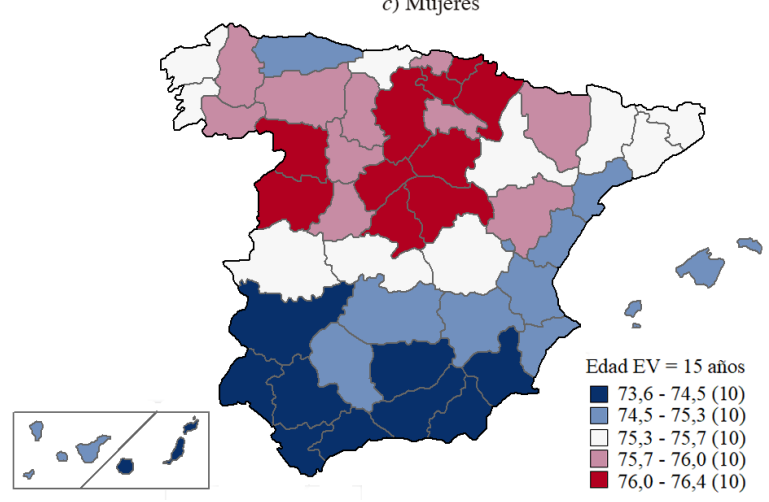

Fuente: elaboración propia a partir de las proyecciones del INE, específicamente, los resultados provinciales: serie 2014-2028. Ver sección de datos para más detalle. 
Figura 7. La tasa prospectiva en la vejez (TDPV) en por provincias en España, 2019

a) Total

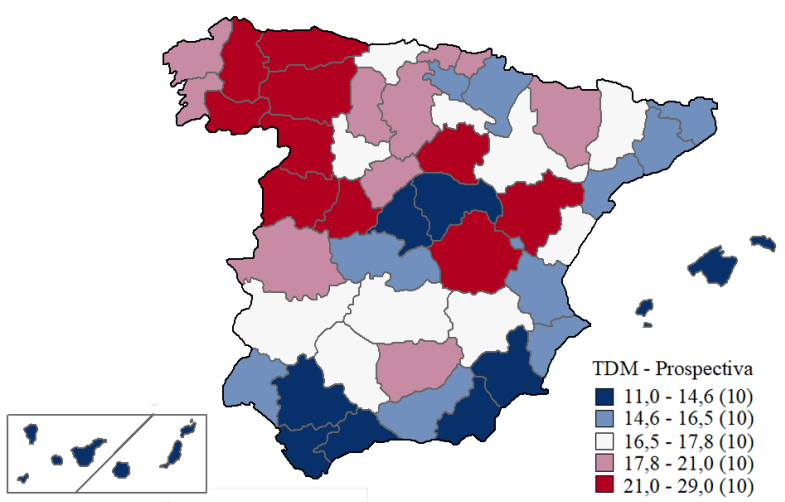

b) Hombres

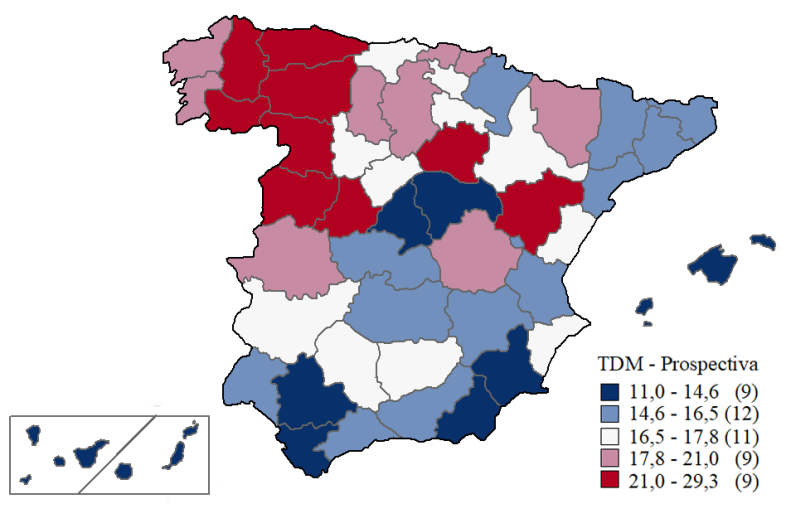

c) Mujeres

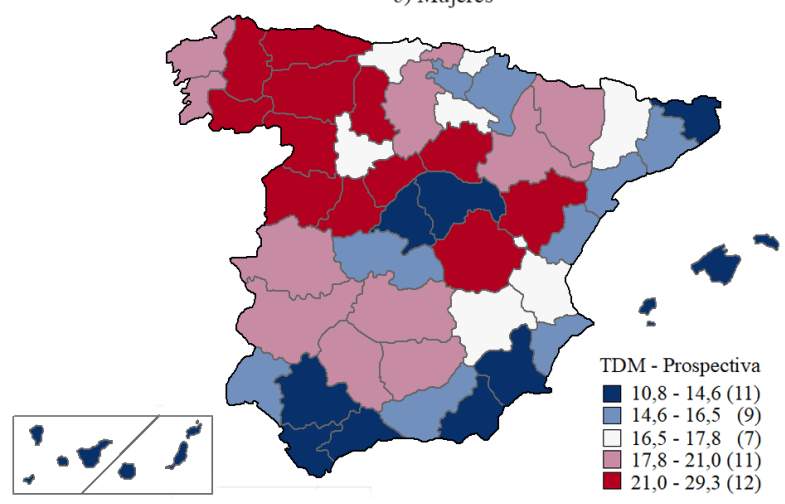

Fuente: elaboración propia a partir de las proyecciones del INE, específicamente, los resultados provinciales: serie 2014-2028. Ver sección de datos para más detalle. 
Figura 8. La tasa de dependencia en la vejez (TDV) por provincias en España, 2019

a) Total

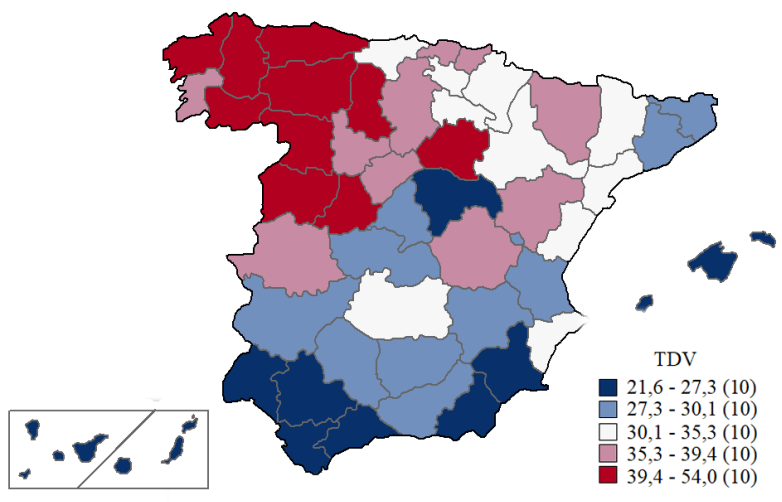

b) Hombres

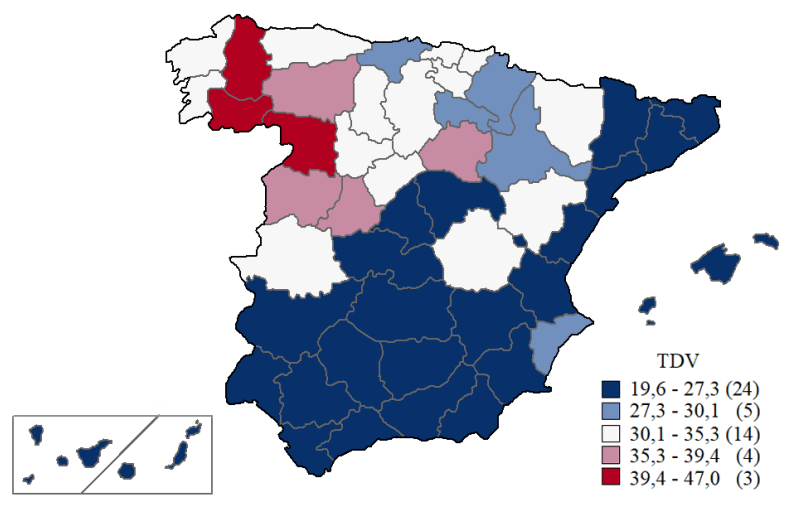

c) Mujeres

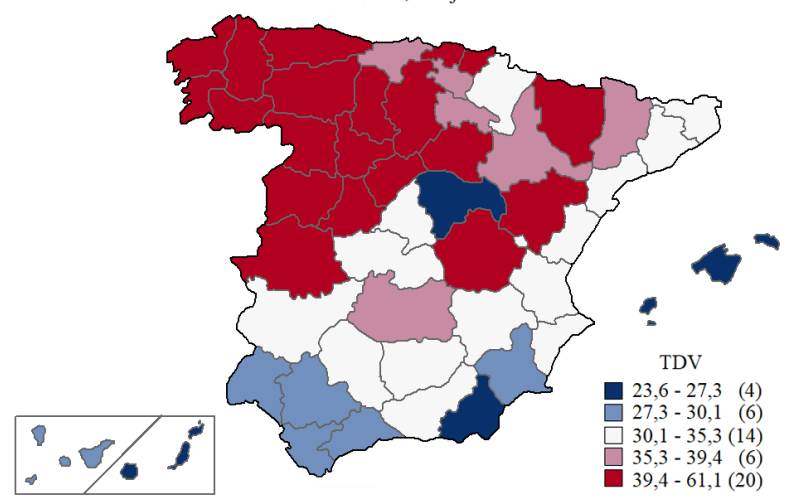

Fuente: elaboración propia a partir de las proyecciones del INE, específicamente, los resultados provinciales: serie 2014-2028. Ver sección de datos para más detalle. 
un hombre de 70 , este dato hace replantear la idea de que hombres y mujeres se consideren igual de envejecidos a la misma edad cronológica, cuando las mujeres tienen mucha más vida por delante, el motivo por la cual este artículo presenta alternativos. También cabe mencionar que las diferencias territoriales son impactantes e importantes. La ratio entre personas mayores y personas en edad laboral (según ambas definiciones del numerador y denominador) es algo más del doble en Orense y Zamora que en las islas Baleares, Guadalajara y Madrid.

El segundo índice alternativo a la TDV es la tasa real de dependencia en la vejez (TRealDV), que controla según el número de personas en empleo. Al considerar los trabajadores que cotizan y contribuyen económicamente a mantener el sistema del bienestar, esta tasa se aproxima mucho más a la realidad que una tasa cuyo denominador incluye todos los adultos en edades laborales o que tienen una EVR de más de 15 años (en el caso de la TPDV). Aunque es la tesorería estatal la que financia las pensiones, un análisis por provincias permite tener una visión más concreta de las regiones donde hay un mejor o peor equilibrio entre los efectivos de personas mayores y los contribuyentes (figura 9). Se debe mencionar que, igual que la TDV, la TRealDV no contabiliza los esfuerzos que se hacen en el entorno informal para, por ejemplo, cuidar a las personas mayores. Por otro lado, un valor más alto de TRealDV que la media de España significaría que la provincia tiene menos cotizantes por cada persona que está por encima de la edad umbral de la vejez. Tal como se puede observar, hay unas diferencias notables entre el patrón territorial de TRealDV y el de las figuras anteriores (al comparar los mapas para la población total ${ }^{5}$ ). Algunas de las provincias con tasas muy altas de paro, sobre todo las del sur, como Cádiz y Huelva, tienen una TRealDV menos favorable con relación a otras provincias con tasas de paro más moderadas, como el País Vasco y la Rioja, donde la TRealDV es relativamente más favorable que la TPDV.

La figura 10 muestra el nivel del paro (cifras oficiales) y la figura 11 la proporción de inactivos (pero tomando el mismo denominador como el de la TPDV en vez de la edad 15-64). Observando con más detalle, no es tanto que las provincias con altos niveles de paro o inactivos tengan altos niveles de TRealDV, sino que, si tuvieran una tasa de paro o de inactividad inferior, la TRealDV hubiera sido relativamente más baja en estas provincias ya que el nivel de TRealDV también está relacionado con el tamaño de la población en edades laborales (ver fórmula 3). Esa es la razón por la que, por ejemplo, Asturias, tiene una TRealDV muy alta, y por la que, aunque con un paro del 14\%, estuviera alrededor de la media nacional en 2019. También destacan Madrid, Guadalajara y Navarra, que con una edad en la que la EVR es igual a 15 años es muy alta (74+ años) y un nivel de paro relativamente bajo $(<12,5 \%)$ tienen una tasa TRealDV también muy baja. En cambio, los bajos niveles de paro en Lugo y La Coruña no han podido compensar el alto nivel

5. Para simplificar y no entrar en polémica, solo se ha calculado este indicador por la población total y no por sexo. 
Figura 9. La tasa real de dependencia de la vejez (TRealDV) por provincias en España, 2019

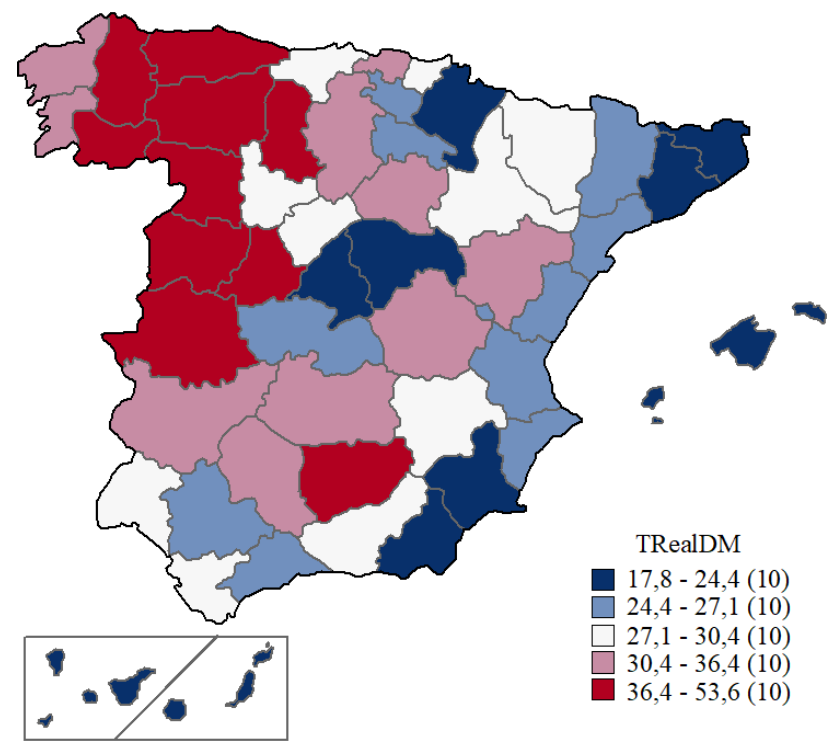

Fuente: elaboración propia a partir de las proyecciones del INE, específicamente, los resultados provinciales: serie 2014-2028 y la EPA. Ver sección de datos para más detalle.

Figura 10. Nivel de paro en España, 2019

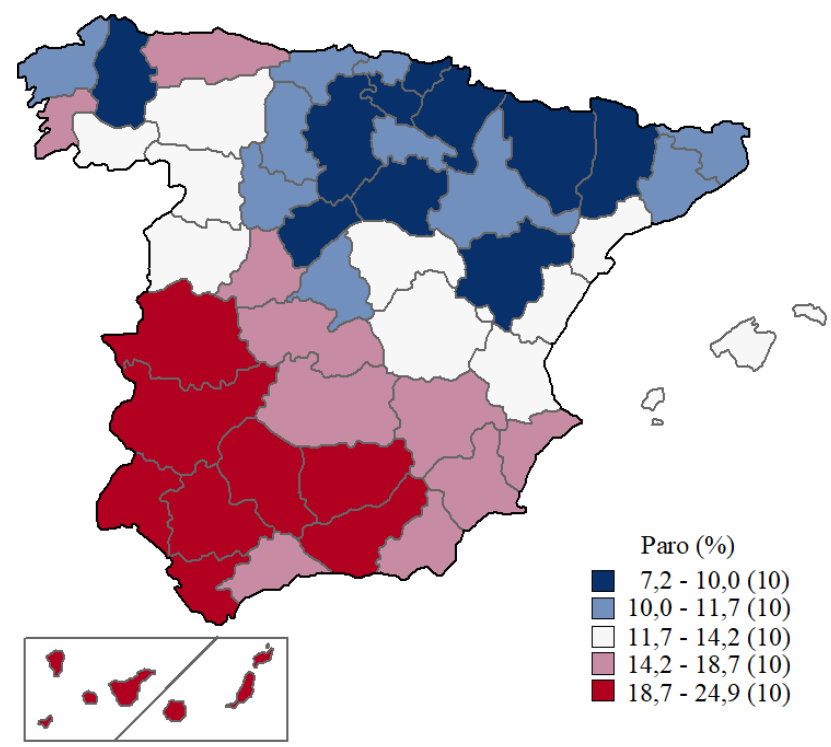

Fuente: elaboración propia a partir de las proyecciones del INE, específicamente, los resultados provinciales: serie 2014-2028 y la EPA. Ver sección de datos para más detalle. 
Figura 11. Proporción inactiva en España, 2019

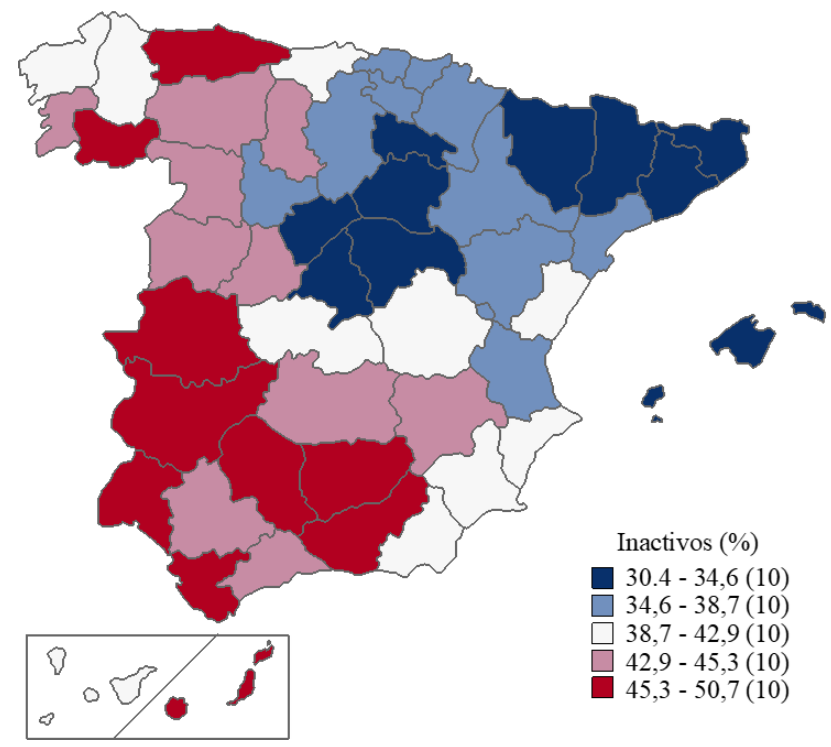

Nota: El denominador en la figura 11 es la población de 20+ años que tiene una EVR de $\geq 15$ años.

Fuente: elaboración propia a partir de las proyecciones del INE, específicamente, los resultados provinciales: serie 2014-2028 y la EPA. Ver sección de datos para más detalle.

de envejecimiento. Teniendo en cuenta también las demás personas inactivas (figura 11), el patrón territorial es bastante similar al del paro, aunque todas las provincias en el noreste del país están peor situadas. La razón es porque, aunque los niveles de paro son (relativamente) bajos, estas provincias tienen proporciones relativamente altas de personas inactivas que no participan en el mercado laboral, en parte por la estructura de edad, es decir, que tienen entre 50 y la edad donde la EVR es igual a 15 años (71-76 años).

\section{Discusión}

El nivel y la tendencia de indicadores alternativos mostrados aquí son muy diferentes que los que indican la tasa de dependencia en la vejez (TDV). Si tenemos en cuenta las mejoras en la esperanza de vida, la tasa de dependencia prospectiva en la vejez (TPDV) es casi igual en la actualidad que en 1950. Si además ajustamos paulatinamente por mejoras en la tasa de actividad a niveles de Suecia (es decir, acercar a la utilización de la capacidad productiva máxima del país) y un aumento en la participación laboral de personas mayores, la tasa real de dependencia en la vejez (TRealDV) no cambiaría en absoluto entre hoy y 2031. No es un resultado fabricado: según cálculos hechos por Fernández Cordón (2017), que hace una suposición similar de mejoras en la tasa de empleo durante las próximas décadas, pero solo para las 
edades 15-64, la ratio de dependencia total (cualquier edad) sobre ocupados (RDTO) no cambiaría casi nada durante todo el periodo de su proyección (2016-2066).

Reconsiderar el envejecimiento puede servir para proporcionar tanto a los políticos como al público en general una visión más realista de la dependencia en edades avanzadas. De hecho, el envejecimiento se basa en una historia optimista sobre una mayor esperanza de vida y una mejor salud. La esperanza de vida restante (EVR) da una visión más exacta del grado de envejecimiento al tener en cuenta el descenso de los riesgos de mortalidad a edades avanzadas, por lo cual se utiliza un umbral de edad variable, definido por una situación de dependencia constante, y no un límite de edad constante.

Durante el proceso de envejecimiento de la población, la fuerza de trabajo se concentra cada vez más en los grupos de mayor edad, ya que generalmente uno es consciente de su propio riesgo de mortalidad (Hamermesh, 1985; Post y Hanewald, 2012), sabe que pasará más años inactivos que las generaciones anteriores una vez que se haya jubilado. Esto puede ser un fuerte incentivo para que la gente acumule activos para su retiro. También es importante para los políticos darse cuenta de que ya no se pueden comparar personas de 65 años en diferentes momentos del tiempo. Por lo tanto, en un contexto histórico esperaríamos que la definición de la «tercera edad» y de «dependiente» fuese bastante diferente (razón por la que a principios de siglo surgió la idea de una «cuarta edad», representada por los «mayores enfermizos»; Gilleard y Higgs, 2011; Baltes y Smith, 2003).

La medición de la TPDV representa tal vez una «cota superior» de optimismo sobre la potencial liberación de capital humano mediante la optimización de las experiencias de la población de más de 65 años que todavía tenga una EVR mayor de 15 años, población que actualmente se describe incorrectamente como «vieja» o «dependiente» (Spijker y Schneider, 2020). Se puede objetar sustituir un umbral fijo (65 años) por otro que también es fijo (la edad donde la $E V R=15$ años), pero en este nuevo umbral la edad es dinámica y se basa en la suposición de que la discapacidad y la «dependencia» se concentran básicamente en los últimos 15 años de vida (Lafortune y Balestat, 2007; Sanderson y Scherbov, 2007). Además, la creciente bibliografía sobre la compresión de la morbilidad (Liu et al., 2009) sugiere que dicho umbral de dependencia es razonable. Dicho eso, para futuras investigaciones, una mejora que se podría contemplar en relación con los indicadores mostrados aquí es distinguir entre la población mayor que está en buena salud y la que está en mala salud (ver también Blanes y Cabré (2015) y Spijker (2015: indicadores 10-16)). Este tipo de indicadores podrían tener utilidad sobre todo para proyectar gastos sanitarios. Es bien sabido que la mayoría de los gastos de atención médica aguda se concentran en las etapas finales de la vida, sin apenas importar la edad en la que se producen (Miller, 2001; Payne et al., 2007). Teniendo en cuenta el rápido ritmo de envejecimiento de la población y una situación económica delicada en España, podría ser de gran utilidad formular políticas económicas, sociales y de sanidad basadas en indicadores más adecuados sobre el nivel y la carga 
económica del envejecimiento en la población, como la TPDV y la TRealDV $\mathrm{u}$ otros que tienen en cuenta la EVR y/o la actividad laboral cambiante.

Los resultados presentados en este breve estudio muestran que la edad con un nivel de EVR igual a 15 años aumentó de forma constante en España desde los años 1950, lo que limita el incremento de la ratio de dependencia prospectiva (TPDV). Aunque la tendencia es parecida en otros países desarrollados, España sigue siendo uno de los más envejecidos. Es por eso que, dentro del debate global sobre el envejecimiento de la población, España merece una atención especial, porque el impacto y las consecuencias del envejecimiento parecen estar exacerbados por su bajísimo nivel de fecundidad (lo que significa que en el futuro habrá menos población activa por cada persona anciana), una altísima esperanza de vida de los ancianos (la mayoría de los recién jubilados tienen una EVR superior a 20 años) y unos niveles altos de inactividad (que reducen los ingresos públicos y, por ende, el gasto en salud y las prestaciones sociales). No obstante, la investigación en el tema del envejecimiento tendría que tener una perspectiva más equilibrada de sus causas y consecuencias, y no considerarse siempre como una catástrofe potencial (Pérez Díaz, 2010; Fernández Cordón, 2015).

La viabilidad del estado de bienestar y del sistema de salud público está más relacionada con la participación laboral, el nivel de productividad y el sistema de tributación de un país que con el envejecimiento demográfico, al menos a corto y medio plazo (Blanes y Cabré, 2015; Fernández Cordón, 2015, 2017; AIReF, 2019), sobre todo, cuando el consumo de las personas mayores está financiado principalmente por el Estado a través de las transferencias públicas (por ejemplo, pensiones del Estado), como es el caso de España. Por tanto, las respuestas económicas y políticas al envejecimiento de la población y a la disminución de la población en edad laboral son la clave para el continuo crecimiento económico de un país.

El aumento gradual de la edad ordinaria de retiro a los 67 años en 2027 ha sido una de esas respuestas (e, indirectamente, un reconocimiento de los aumentos en EVR en la vejez), pero el Gobierno también debe tener en cuenta el potencial de la población laboral (ver figura 2). Bajar el desempleo del 15\% en la actualidad (solo por delante de Grecia) a niveles de los países nórdicos, de habla alemana, del Benelux y el Reino Unido (entre el 3\% y el $7 \%$ en 2018) (<https://ec.europa.eu/eurostat/web/lfs/data/database >), subir la baja tasa de ocupación laboral de personas entre 50 y 64 años y bajar el alto nivel de actividad económica «informal» (aproximadamente, el 18\% del PIB nacional en 2015) (Schneider, 2016) son acciones que mejorarían en mayor medida las arcas públicas que subir la edad de jubilación. Además, la última medida mencionada — subir la edad de jubilación — es injusta desde un punto de vista social: las clases más desfavorecidas perderán una proporción mayor de la jubilación que los ricos, ya que tienen una menor esperanza de vida (Martín et al., 2019), salvo si se ajusta la edad de jubilación según el nivel de EVR de la clase social o la rama de actividad a la que pertenece una persona. No obstante, a más largo plazo, desde mediados de la próxima década en adelante, 
una mayor participación laboral, también en edades cercanas a la edad legal de jubilación, no podría compensar la reducción de la población en edad activa, o sea, el efecto del factor demográfico. Por lo tanto, el coste del aumento en la esperanza de vida en edades avanzadas recaerá sobre el ahorrador, que tendrá que elegir entre cobrar menos pensión o retrasar su jubilación, dos opciones actuarialmente equivalentes (Fernández Cordón, 2015).

Gestionar las consecuencias del envejecimiento de la población no es de ninguna manera solo un asunto nacional. Aunque las pensiones y una parte importante de la salud pública están atendidas por el Estado, analizar el grado de envejecimiento según las regiones puede dar indicaciones sobre donde se debe prestar más atención a la hora buscar vías para gestionar mejor los retos del envejecimiento. Por ejemplo, tal como demuestra la figura 7, Madrid, sus alrededores y la costa sur y Mediterránea están mejor situados que la mayoría de las provincias de Galicia y Castilla y León, porque tienen por cada anciano relativamente más adultos por debajo del umbral de vejez. Sin embargo, las provincias andaluzas y extremeñas pierden esa ventaja cuando se consideran solo las personas que trabajan (figura 8), debido a los niveles más altos de desempleo e inactividad económica. Si bien, por un lado, esto significa menos ingresos fiscales para el Estado que pueden destinarse a las pensiones estatales, el sistema de salud pública y el Sistema para la Autonomía y Atención a la Dependencia; por otro lado, hay más personas disponibles para el cuidado informal de las personas mayores, algo que en el caso de España sigue recayendo mayoritariamente en las manos de la familia (Spijker y Zueras, 2016). Dicho eso, tal como explica Fernández Cordón (2015), el cambio demográfico en curso no se traduce en un aumento insostenible de la carga sobre los activos por una reducción en los recursos, sino en la dificultad que supone modificar la distribución actual de estos recursos para adaptarla al cambio radical del tipo de dependientes que será necesario atender (más personas mayores, menos niños $\mathrm{y}$ adultos dependientes).

De cara a investigaciones futuras, sería deseable medir los años de vida restante y la participación laboral desde un enfoque generacional, a diferencia del método estándar basado en la evolución cronológica de los indicadores. Ese enfoque, a pesar de las dificultades que conllevan los elevados requisitos de información, permitiría apreciar con mayor claridad la magnitud de los cambios en el ciclo de vida de los individuos y, por lo tanto, planificar mejor las necesidades de atención médica y pensiones de las futuras generaciones de personas mayores.

Para terminar, la población mayor es muy diversa y está experimentando mejoras en la salud y en sus recursos económicos, lo que la hace más independiente y activa. Por eso también deberíamos acabar con la práctica de aplicar definiciones rígidas, usar una edad fija para referirnos a este colectivo (por ejemplo, mayor de 65 años) o poner etiquetas como «dependiente»o «improductivo» a las personas mayores, ya que muchos ni son dependientes ni improductivos, sobre todo en términos no económicos (Spijker y Schneider, 2020). Utilizar un umbral de dependencia como la edad donde la esperanza 
de vida restante es igual o inferior a los 15 años, que varía en el tiempo por los cambios en la esperanza de vida, y tener en cuenta los cambios en la fuerza del trabajo son dos posibles factores a valorar a la hora de definir indicadores que cuantifiquen el nivel de dependencia de las personas «mayores» en una población. Debido al uso generalizado de indicadores inadecuados de envejecimiento de la población, tales como la tasa de dependencia de la vejez, este fenómeno es a menudo visto como una amenaza para el crecimiento económico y los presupuestos públicos, a pesar de que muchos otros factores están implicados en los incrementos observados y proyectados de los gastos del sistema sanitario y de las prestaciones sociales, o de la producción económica. De hecho, una población que envejece incluso tiene un gran potencial de crecimiento económico con las políticas correctas (Mason y Lee, 2006). Sin embargo, es importante que se haga un uso más generalizado de indicadores de envejecimiento de la población más precisos, para que el público, los responsables de las políticas públicas e incluso académicos puedan entender el lado positivo del envejecimiento.

\section{Referencias bibliográficas}

AIReF (Autoridad INDEPENDIENTE De Responsabilidad FisCAL) (2019). «Opinión sobre sostenibilidad del sistema de Seguridad Social». Recuperado de <https:// www.airef.es/wp-content/uploads/2019/01/opinion-pensiones/190109_Opinion_SS.pdfs.

Baltes, P. B. y Smith, J. (2003). "New Frontiers in the Future of Aging: From Successful Aging of the Young Old to the Dilemmas of the Fourth Age». Gerontology, 49, 123-135. $<$ https://doi.org/10.1159/000067946>

Berger, N. y Van Oyen, H. (2013). «Work Package 7 GALI validation using the European Health Interview Survey». EHLEIS Technical report 2013_7.1. Recuperado de <http://www.eurohex.eu/pdf/Reports_2013/2013_TR7\%201_Gali\%20 validation.pdf $>$.

Bíró, A. (2013). «Subjective mortality hazard shocks and the adjustment of consumption expenditures». Journal of Population Economics, 26 (4), 1.379-1.408.

Blanes, A. (2007). La mortalidad en la España del siglo XX. Análisis demográfico y territorial. Tesis doctoral. Universitat Autònoma de Barcelona.

BLANES, A. y CABRÉ, A. (2015). «Inercias e incertidumbres en el futuro demográfico de España». Revista del Ministerio de Empleo y Seguridad Social, 119, 105-129.

Blanes, A.; Gil, F. y Pérez DíAz, J. (1996). «Población y actividad en España: evolución y perspectivas». Colecciones, Estudios e Informes 5: La Caixa. Recuperado de $<$ https://digital.csic.es/bitstream/10261/5581/1/PerezEsp1996.pdf>.

Brouard, N. (1989). Mouvements et modeles de population. Yaoundé, Camerún: Institut de Formation et de Recherche Démographiques. Recuperado de <http://sauvy. ined.fr/brouard/enseignements/iford/mouvementetmodeles.pdf>.

CARSTENSEN, L. L. (2006). «The influence of a sense of time on human development». Science, 312 (5.782), 1.913-1.915.

Carstensen, L. L.; Isaacowitz, D. M. y Charles, S. T. (1999). «Taking time seriously: A theory of socioemotional selectivity». American Psychologist, 54 (3), 165. 
Cocco, J. F. y Gomes, F. J. (2012). «Longevity risk, retirement savings, and financial innovation». Journal of Financial Economics, 103 (3), 507-529.

Doyle, Y.; McKeE, M.; Rechel, B. y Grundy, E. (2009). "Meeting the challenge of population ageing». BMJ 339 (b3926), 892-894.

Fernández Cordón, J. A. (1996). Demografía, actividad y dependencia en España. Bilbao: Fundación BBV-Documenta.

- (2015). «Relaciones intergeneracionales, demografía y economía en relación con las pensiones». Cuadernos de Relaciones Laborales, 33 (2), 235. <https://doi.org/10.5209/rev_CRLA.2015.v33.n2.50315>

- (2017). «De los cambios demográficos a la economía del cuidado». Gaceta Sindical: Reflexión y Debate, 28, 65-82.

Gilleard, C. y Higgs, P. (2011). «Frailty, disability and old age: A re-appraisal». Health, 15 (5), 475-490.

Hamermesh, D. S. (1985). «Expectations, life expectancy, and economic behavior». The Quarterly Journal of Economics, 100 (2), 389-408.

IMF (2020). World Economic Outlook Database (April 2020). Recuperado de <https:// www.imf.org/en/Publications/WEO/Issues/2020/04/14/weo-april-2020>.

Lafortune, G. y Balestat, G. (2007). «Trends in Severe Disability Among Elderly People: Assessing the Evidence in 12 OECD Countries and the Future Implications». En: OECD Health Working Papers. OECD Publishing.

LiU, J.; Chen, G.; Song, X.; ChI, I. y Zheng, X. (2009). «Trends in disability-free life expectancy among Chinese older adults». Journal of Aging and Health, 21 (2), 266-285.

Lutz, W.; SAnderson, W. y Scherbov, S. (2008). «Global and Regional Population Ageing: How Certain Are We of its Dimensions?». Journal of Population Ageing, 1 (1), 75-97. $<$ https://doi.org/10.1007/s12062-009-9005-5>

Maré, D. C. (2018). «The labor market in New Zealand, 2000-2017». IZA World of Labor (427). <https://doi.org/10.15185/izawol.427>

Martín, U.; Domínguez-RodrígueZ, A. y Bacigalupe, A. (2019). «Desigualdades sociales en salud en población mayor: una aportación desde la salud pública al debate sobre el retraso de la edad de jubilación en España». Gaceta Sanitaria, 33, 82-84.

Mason, A. y LeE, R. (2006). «Reform and support systems for the elderly in developing countries: capturing the second demographic dividend». Genus, 62 (2), 11-35.

Miller, T. (2001). "Increasing longevity and Medicare expenditures». Demography, 38 (2), 215-226.

OECD (2019). Revenue Statistics. París: OECD Publishing.

- (2020). «Labour Market Statistics: Labour force statistics by sex and age: indicators». OECD Employment and Labour Market Statistics (database). <https://doi.org/10.1787/data-00310-en>

Payne, G.; Laporte, A.; Deber, R. y Coyte, P. C. (2007). "Counting Backward to Health Care's Future: Using Time-to-Death Modeling to Identify Changes in End-of-Life Morbidity and the Impact of Aging on Health Care Expenditures». The Milbank Quarterly, 85 (2), 213-257. <https://doi.org/10.1111/j.1468-0009.2007.00485.x>

PÉreZ DÍAZ, J. (2010). «Demografía. El envejecimiento de la población española». Investigación y Ciencia: Edición Española de Scientific American, 410, 34-42. 
Polder, J. J.; Bonneux, L.; MeErding, W. J. y MaAs, P. J. van der (2002). «Age-specific increases in health care costs». European Journal of Public Health, 12 (1), 57-62.

Post, T. y HANEWALD, K. (2012). «Longevity risk, subjective survival expectations, and individual saving behavior». Journal of Economic Behavior \& Organization, 86, 200-220.

RIVERO-CANTILlANO, R. y SPIJKER, J. (2015). «Del rejuvenecimiento al envejecimiento de la población ¿̨o viceversa?: Chile en el contexto de América Latina, 1950-2050». Notas de Población, 101, 127-156.

Ryder, N. B. (1975). "Notes on Stationary Populations». Population Index, 41 (1), $3-28$.

SANDERSON, W. C. y Scherbov, S. (2005). «Average remaining lifetimes can increase as human populations age». Nature, 435 (7.043), 811-813.

- (2007). "A new perspective on population aging». Demographic Research, 16 (2), 27-58.

- (2010). «Remeasuring Aging». Science, 329 (5.997), 1.287-1.288. <https://doi.org/10.1126/science.1193647>

SCHNEIDER, F. (2016). "Estimating the size of the shadow economies of highlydeveloped countries: selected new results». CESifo DICE Report, 14 (4), 44-53.

SPIJKER, J. (2015). "Alternative indicators of population ageing: An inventory». Vienna Institute of Demography Working Papers, 4/2015. Vienna Institute of Demography.

SPIJKER, J. y MACINNES, J. (2013). «Population ageing: the timebomb that isn't?». British Medical Journal, 347. <https://doi.org/10.1136/bmj.f6598>

SPIJKER, J. y SCHNEIDER, A. (2020). "The myth of old age: Addressing the issue of dependency and contribution in old age using empirical examples from the UK». Sociological Research Online. <https://doi.org/10.1177/1360780420937737>

SPIJKER, J. y Zueras, P. (2016). "El cuidado a los mayores en un contexto de envejecimiento, cambio social, político y económico». Panorama Social, 23, 167-182.

The Conference Board (2019). "The Conference Board Total Economy Database (adjusted version)». Recuperado de <http://www.conference-board.org/data/economydatabase/ $>$.

The ECONOMIst InTELligenCE UNIT (2020). «Spain. Economy: Long-term outlook». Recuperado de <https://country.eiu.com/spain\#>.

VAn Solinge, H. y Henkens, K. (2010). «Living longer, working longer? The impact of subjective life expectancy on retirement intentions and behaviour». European Journal of Public Health, 20 (1), 47-51.

Vaupel, J. W. (2009). "Life lived and left: Carey's equality». Demographic Research, $20(3), 7-10$.

Villavicencio, F. y Riffe, T. (2016). «Symmetries between life lived and left in finite stationary populations». Demographic Research, 35, 381-398.

Wolf, D. A. y Amirkhanyan, A. A. (2010). «Demographic Change and Its Public Sector Consequences». Public Administration Review, 70, 12-23. <https://doi.org/10.1111/j.1540-6210.2010.02242.x>

Wong, A.; BaAl, P. H. M. van; Boshuizen, H. C. y Polder, J. J. (2011). «Exploring the influence of proximity to death on disease-specific hospital expenditures: a carpaccio of red herrings». Health Economics, 20 (4), 379-400. 
